\title{
DESIGNING (IN)FINITE-ALPHABET SEQUENCES VIA SHAPING THE RADAR AMBIGUITY FUNCTION
}

\author{
Mohammad Alaee-Kerahroodi ${ }^{\star}$,Saeid Sedighi, Bhavani Shankar M.R. and Björn Ottersten \\ Interdisciplinary Centre for Security, Reliability and Trust (SnT), University of Luxembourg \\ *mohammad.alaee@uni.lu
}

\begin{abstract}
In this paper, a new framework for designing the radar transmit waveform is established through shaping the radar Ambiguity Function (AF). Specifically, the AF of the phase coded waveforms are analyzed and it is shown that a continuous/discrete phase sequence with the desired AF can be obtained by solving an optimization problem promoting equality between the $\mathrm{AF}$ of the transmit sequence and the desired AF. An iterative algorithm based on Coordinate Descent (CD) method is introduced to deal with the resulting non-convex optimization problem. Numerical results illustrate the proposed algorithm make it possible to design sequences with remarkably high tolerance towards Doppler frequency shifts, which is of interest to the future generations of automotive radar sensors.
\end{abstract}

Index Terms - Ambiguity Function, Coordinate Descent, Radar, Waveform Design.

\section{INTRODUCTION}

Emerging automotive radar sensors, operating at $79 \mathrm{GHz}$ frequency and occupying $4 \mathrm{GHz}$ bandwidth, aim to enhance safety for occupants and pedestrians alike [1-6]. The higher bandwidth while providing higher range resolution, also leads to lower Signal to Noise Ratio (SNR) as additional noise components are incorporated in the received signal. This, coupled with limited transmit power and conservative link budgets necessitates enhancement of SNR using appropriate matched filtering of the received signal $[7,8]$.

Ideally, for a pulse compression radar system, the matched filter achieves to the highest possible SNR when there is no Doppler frequency offset in the target reflected signal [7-9] or when the Doppler shift is compensated in the processing unit using the mismatched filter [10]. If neither is the case, a Doppler mismatch loss is usually experienced $[7,8]$. Consequently, radar engineers usually keep an eye on the AF of the waveforms

This work is partially supported by the Luxembourg National Research Fund under the BRIDGES project "Adaptive mm-Wave Radar platform for Enhanced Situational Awareness: Design and Implementation (AWARDS)" as well as AFR grant for bilateral Ph.D. project (Project Reference 11228830) on "Compressive Sensing for Ranging and Detection in Automotive Applications". to assess the loss incurred due to the different Doppler shifts or delays of the targets [11-14].

In classical automotive radar sensors, Frequency Modulated Continuous Wave (FMCW) waveform is employed due to the advantages of large time-bandwidth product (high range resolution) and high Doppler tolerance $[3,7,12]$. However, as the number of vehicles with radar systems increases, the probability of radar signal interference and the accompanying ghost target problems become serious. In classical FMCW waveforms this has been solved in using multiple chirp signals with different slope [3]. But in this case, a long measurement time is needed which is a contradiction to a high update rate. An alternative solution is to use Code Division Multiplexing (CDM) $[15,16]$ to efficiently make the orthogonality between different transmit sequences, provided that unknown Doppler shift of the targets does not degrade the SNR $[12,17]$. SNR robustness to Doppler can be enhanced through code design and Frank, Golomb, P1, P2, P3, P4, etc., are some examples, which are unfortunately constrained to the specific lengths $[9,11]$.

In this paper, we consider the problem of shaping $\mathrm{AF}$, typically such that we can design a continuous/discrete phase sequence with high Doppler tolerance properties. The works most similar to this paper are [11-14], where $L$-phase ${ }^{1}$ unimodular sequences [11], Linear-FM Synthesized (LFM-Syn) waveform [12], slow-time constant modulus sequences [13], and phase-only modulated waveforms [14] are proposed to shape the AF. However, none of these algorithms tackle the non-convex optimization problem of shaping AF with discrete alphabet constraint at the design stage. The relevant gap is filled in this paper, by proposing a unified framework to design continuous/discrete phase sequences with desired AF. Note that the pulse compression radar systems can only transmit unimodular sequences since radio frequency power amplifiers have nonlinear relationships between their input and output and they cannot have maximum

\footnotetext{
${ }^{1}$ In [11], the $L$-phase sequence is obtained by choosing the value in the phase set, which is closest to the arbitrary value obtained in the solution of unimodular optimization problem. However, quantizing an optimized sequence does not guarantee to achieve an optimal discrete phase sequence, specifically when the alphabet size is small [18].
} 
power efficiency at all power levels. Also, they typically generate phase sequences in discrete form due to the ease of generation and implementation $[7,8]$.

The remainder of this paper is organized as follows. Section 2, defines the cost function for shaping the AF. Section 3 provides the solution of the non-convex constrained optimization problem. Simulation results are provided in Section 4 and Section 5 concludes the paper.

\section{PROBLEM FORMULATION}

Let $\boldsymbol{x}=\left[x_{1}, x_{2}, \ldots, x_{N}\right]^{T} \in \mathbb{C}^{N}$ be the transmitted unimodular $^{2}$ sequence where $N$ is the intra-pulse code length. The discrete radar AF is expressed by $[11,12]$ :

$$
|\chi(m, \nu)|=\left|\sum_{n=1}^{N-m} x_{n}^{*} x_{n+m} e^{-\jmath \frac{2 \pi \nu}{N}(n+m)}\right|,
$$

where $m, \nu \in\{1-N, \ldots, N-1\}$ denote the time and Doppler-shift indices, respectively. Indeed, $m$ and $\nu$ are normally associated with target range, and Doppler shift, respectively. In addition, let $\boldsymbol{c}(\boldsymbol{x}, \boldsymbol{y})$ denote the aperiodic cross-correlation of sequences $\boldsymbol{x}$ and $\boldsymbol{y}=\left[y_{1}, y_{2}, \ldots, y_{N}\right]^{T} \in \mathbb{C}^{N}$, with

$$
\begin{aligned}
\boldsymbol{c}(\boldsymbol{x}, \boldsymbol{y}) & =\left[c_{1-N}(\boldsymbol{x}, \boldsymbol{y}), \ldots, c_{0}, \ldots, c_{N-1}(\boldsymbol{x}, \boldsymbol{y})\right]^{T}, \\
c_{m}(\boldsymbol{x}, \boldsymbol{y}) & =\sum_{n=1}^{N-m} x_{n}^{*} y_{n+m},
\end{aligned}
$$

defining $\boldsymbol{y}^{\nu}=\left[x_{1} e^{-\jmath \frac{2 \pi \nu}{N}}, x_{2} e^{-\jmath \frac{4 \pi \nu}{N}}, \ldots, x_{N} e^{-\jmath 2 \pi \nu}\right]^{T} \in$ $\mathbb{C}^{N}$, results

$$
|\chi(m, \nu)|=\left|c_{m}\left(\boldsymbol{x}, \boldsymbol{y}^{\nu}\right)\right|
$$

Equation (2) indicates the AF can be interpreted as the matched filter output for stationary targets if $\nu=0$ (matched-Doppler, range cut of the AF).

Let the modulus of a desired AF (non-negative) be given by $\left|\chi_{D}(m, \nu)\right|$. A design objective would be to minimize the following squared error between the desired and generated $\mathrm{AF}$

$$
J(\boldsymbol{x})=\left.\sum_{m=1-N}^{N-1} \sum_{\nu=1-N}^{N-1}|| \chi(m, \nu)\right|^{2}-\left.\left|\chi_{D}(m, \nu)\right|^{2}\right|^{2} .
$$

The optimization can then be cast as,

$$
P_{\boldsymbol{x}}^{\infty}=\left\{\begin{array}{ll}
\min _{\boldsymbol{x}} & J(\boldsymbol{x}) \\
\text { s.t. } & \boldsymbol{x} \in \Omega_{\infty}
\end{array}, \quad P_{\boldsymbol{x}}^{L}=\left\{\begin{array}{cl}
\min _{\boldsymbol{x}} & J(\boldsymbol{x}) \\
\text { s.t. } & \boldsymbol{x} \in \Omega_{L}
\end{array}\right.\right.
$$

where the constraints $\boldsymbol{x} \in \Omega_{\infty}$, and $\boldsymbol{x} \in \Omega_{L}$ identify continuous alphabet ${ }^{3}$, and finite alphabet codes, respectively. Precisely, $\Omega_{\infty}=\left\{\boldsymbol{x} \in \mathbb{C}^{N}|| x_{n} \mid=1, n=\right.$

\footnotetext{
${ }^{2}$ Unimodular sequences are those for which the sequence elements may be of any phase, not constrained to the two states ( 0 and $\pi$ ) of binary sequences.

${ }^{3}$ Continuous phase means the phase values can get any arbitrary value within $[0,2 \pi)$. For the discrete phase, the feasible set is restricted to a finite number of equi-spaced points on the unit circle.
}

$1, \ldots, N\}$ and $\Omega_{L}=\left\{\boldsymbol{x} \mid x_{n} \in \Psi_{L}, n=1, \ldots, N\right\}$, where $\Psi_{L}=\left\{1, \bar{\omega}, \ldots, \bar{\omega}^{L-1}\right\}, \bar{\omega}=e^{j \frac{2 \pi}{L}}$ and $L$ is the size of discrete constellation alphabet.

\section{AF OPTIMIZED WAVEFORMS}

In order to tackle the non-convex constrained problems $P_{\boldsymbol{x}}^{\infty}$ and $P_{\boldsymbol{x}}^{L}$, we use the CD framework, i. e., sequentially optimizing the objective with respect to one variable, keeping the others fixed $[16,18]$. With reference to $(4)$, the optimization problem at step $k+1$ is,

$$
\left\{\begin{array}{ll}
\min _{x_{d}} & J\left(x_{d} ; \boldsymbol{x}_{-d}^{(k)}\right) \\
\text { s.t. } & \left|x_{d}\right|=1
\end{array}, \quad \begin{cases}\min _{x_{d}} & J\left(x_{d} ; \boldsymbol{x}_{-d}^{(k)}\right) \\
\text { s.t. } & x_{d} \in \Omega_{L}\end{cases}\right.
$$

where $x_{d}$ is the variable of optimization, and

$$
\boldsymbol{x}_{-d}^{(k)}=\left[x_{1}^{(k)}, \ldots, x_{d-1}^{(k)}, x_{d+1}^{(k)}, \ldots, x_{N}^{(k)}\right]^{T} \in \mathbb{C}^{N-1}
$$

refers to the remaining code entries which are assumed known from previous iteration. To proceed further, let $\mathbf{I}_{A}(t)$ and $\mathbf{I}_{B}(t)$ denote the indicator functions of sets $A=\{1,2, \ldots, N\}$ and $B=\{-1,-2, \ldots,-N+1\}$ respectively, i.e., $\mathbf{I}_{A}(t)=1$ if $t \in A$, otherwise $\mathbf{I}_{A}(t)=0$.

The cross-correlation function $c_{m}\left(\boldsymbol{x}, \boldsymbol{y}^{\nu}\right)$ with explicit dependence on $x_{d}$ is, $c_{m}^{(k)}\left(\boldsymbol{x}, \boldsymbol{y}^{\nu}\right)=a_{m \nu}^{(k)} x_{d}+b_{m \nu}^{(k)}$, with $a_{m \nu}^{(k)} \triangleq y_{d+m}^{*(k)} \mathbf{I}_{A}(d+m)$, and $^{4}$

$$
b_{m \nu}^{(k)} \triangleq \sum_{\substack{n=1 \\ n \neq d}}^{N-m} x_{n}^{(k)} y_{n+m}^{*(k)} \mathbf{I}_{A}(m+1)+\sum_{\substack{n=-m+1 \\ n \neq d}}^{N} x_{n}^{(k)} y_{n+m}^{*(k)} \mathbf{I}_{B}(m) .
$$

With reference to (2), the optimization problems in (5) can be recast as

$$
\begin{aligned}
& P_{d, \boldsymbol{x}^{(k)}}^{\infty}= \begin{cases}\min _{x_{d}} \sum_{m=1-N}^{N-1} \sum_{\nu=1-N}^{N-1}|| a_{m \nu}^{(k)} x_{d}+\left.b_{m \nu}^{(k)}\right|^{2}-\left.\left|\chi_{D}(m, \nu)\right|^{2}\right|^{2} \\
\text { s.t. } & \left|x_{d}\right|=1\end{cases} \\
& P_{d, \boldsymbol{x}^{(k)}}^{L}= \begin{cases}\min _{x_{d}} \sum_{m=1-N}^{N-1} \sum_{\nu=1-N}^{N-1}|| a_{m \nu}^{(k)} x_{d}+\left.b_{m \nu}^{(k)}\right|^{2}-\left.\left|\chi_{D}(m, \nu)\right|^{2}\right|^{2} \\
\text { s.t. } \quad x_{d} \in \Omega_{L}\end{cases}
\end{aligned}
$$

which are non-convex, constrained optimization problems with the objectives as a function of a complex variable. Denoting by $x_{d}^{\star}$ the optimal solution to $P_{d, \boldsymbol{x}^{(k)}}^{\infty}$ $\left(P_{d, \boldsymbol{x}^{(k)}}^{L}\right)$, the optimized radar code at step $k+1$ is $\boldsymbol{x}^{(k+1)}=\left[x_{1}^{(k)}, \ldots, x_{d-1}^{(k)}, x_{d}^{\star}, x_{d+1}^{(k)}, \ldots, x_{N}^{(k)}\right]^{T}$.

\subsection{Code Entry Design; Continuous Phase}

This subsection is focused on the solution of $P_{d, \boldsymbol{x}^{(k)}}^{\infty}$. As first step toward this goal, we provide the following Lemma that shows the objective function, as a function of $x_{d}=e^{\jmath \phi_{d}}, \phi_{d} \in[0,2 \pi)$, can be expressed as a ratio of quartic polynomials in a real variable.

\footnotetext{
${ }^{4}$ The dependence on the variable $\nu$ in $y_{d+m}^{*(k)}$ is implicit for ease of notation.
} 
Lemma 3.1. Performing the change of variable $\zeta_{d}=$ $\tan \left(\frac{\phi_{d}}{2}\right), P_{d, \boldsymbol{x}^{(k)}}^{\infty}$ can be rewritten as follows

$$
\widetilde{P}_{\zeta_{d}}^{\infty}=\left\{\min _{\zeta_{d}} \frac{\widetilde{\alpha}^{(k)} \zeta_{d}^{4}+\widetilde{\beta}^{(k)} \zeta_{d}^{3}+\widetilde{\gamma}^{(k)} \zeta_{d}^{2}+\widetilde{\mu}^{(k)} \zeta_{d}+\widetilde{\eta}^{(k)}}{\left(\zeta_{d}^{2}+1\right)^{2}}\right.
$$

where $\widetilde{\alpha}^{(k)}, \widetilde{\beta}^{(k)}, \widetilde{\gamma}^{(k)}, \widetilde{\mu}^{(k)}$ and $\widetilde{\eta}^{(k)}$ are real-valued coefficients specified in Appendix A.

\section{Proof. See Appendix A.}

Inspired with the above lemma, due to the special form of the polynomials in $\widetilde{P}_{\zeta_{d}}^{\infty}$, the optimal solution can be obtained by finding the real roots of the first order derivative of the objective function, and evaluating the objective function in these points as well as at $\infty$ (see [18] for more details). Finding the optimal value $\zeta_{d}^{\star}$, we set $\phi_{d}^{\star}=2 \operatorname{atan}\left(\zeta_{d}^{\star}\right)$, and $x_{d}^{\star}=e^{J \phi_{d}^{\star}}$.

\subsection{Code Entry Design; Discrete Phase}

Let us now consider Problem $P_{d, \boldsymbol{x}^{(k)}}^{L}$ and develop an efficient procedure to find its optimal solution exploiting Discrete Fourier Transform (DFT). In terms of $\phi_{d}=$ $\arg \left(x_{d}\right) \in[0,2 \pi)$, the optimization problem at step $k$ can be recast as,

$\widetilde{P}_{d, \phi_{d}}^{L} \begin{cases}\min _{\phi_{d}} & \sum_{m=1-N}^{N-1} \sum_{\nu=1-N}^{N-1}|| a_{m \nu}^{(k)} e^{\jmath \phi_{d}}+\left.b_{m \nu}^{(k)}\right|^{2}-\left.\left|\chi_{D}(m, \nu)\right|^{2}\right|^{2} \\ \text { s.t. } & \phi_{d} \in\left\{0, \frac{2 \pi}{L}, \frac{4 \pi}{L}, \ldots, \frac{2 \pi(L-1)}{L}\right\}\end{cases}$

the following lemma provides a key result to tackle Problem $\widetilde{P}_{d, \phi_{d}}^{L}$.

Lemma 3.2. Let

$$
\boldsymbol{\rho}_{m \nu}^{(k)}(\theta)=\left[\left|a_{m \nu}^{(k)} e^{\jmath \theta_{1}}+b_{m \nu}^{(k)}\right|^{2}, \ldots,\left|a_{m \nu}^{(k)} e^{\jmath \theta_{L}}+b_{m \nu}^{(k)}\right|^{2}\right]^{T},
$$

with $\theta_{i}=\frac{2 \pi(i-1)}{L}, i=1, \ldots, L$, and $\boldsymbol{\rho}_{m \nu}^{(k)}(\theta) \in \mathcal{R}^{L}$, then

$$
\boldsymbol{\rho}_{m \nu}^{(k)}(\theta)=\left|D F T\left[a_{m \nu}^{(k)}, b_{m \nu}^{(k)}, \mathbf{0}_{1 \times(L-2)}\right]^{T}\right|^{2},
$$

where the square modulus is element wise.

Proof. Proof is straight forward and can be obtained with the insight of Appendix C in [18].

Inspired from Lemma 3.2, the discrete phase optimization problem is

$$
\widetilde{P}_{d, \theta}^{L}\left\{\left.\left.\min _{\theta} \sum_{m=1-N}^{N-1} \sum_{\nu=1-N}^{N-1}\left|\boldsymbol{\rho}_{m \nu}^{(k)}(\theta)-\right| \chi_{D}(m, \nu)\right|^{2}\right|^{2},\right.
$$

and the optimal solution to $\widetilde{P}_{d, \theta}^{L}$ is given by $\phi_{d}^{\star}=$ $\frac{2 \pi\left(i^{\star}-1\right)}{L}$, where

$i^{\star}=\left.\left.\arg \min _{i=1, \ldots, L} \sum_{m=1-N}^{N-1} \sum_{\nu=1-N}^{N-1}\left|\boldsymbol{\rho}_{m \nu}^{(k)}(\theta)-\right| \chi_{D}(m, \nu)\right|^{2}\right|^{2}$.

Hence, the optimal phase code entry can be efficiently computed as $x_{d}^{\star}=e^{\jmath \phi_{d}^{\star}}$ using DFT.

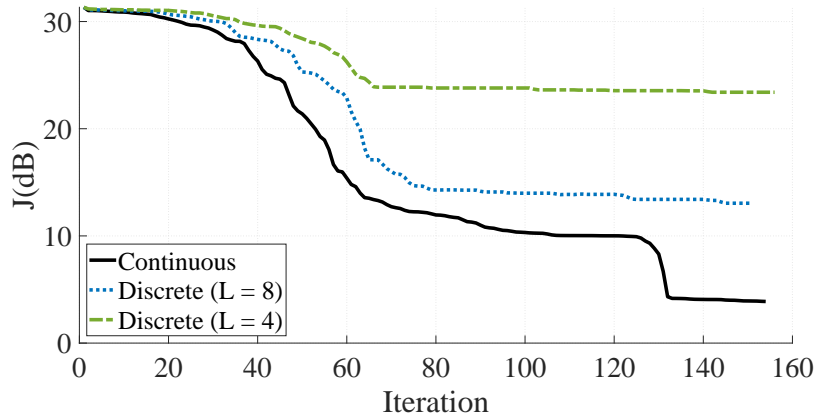

Fig. 1: Convergence behavior.

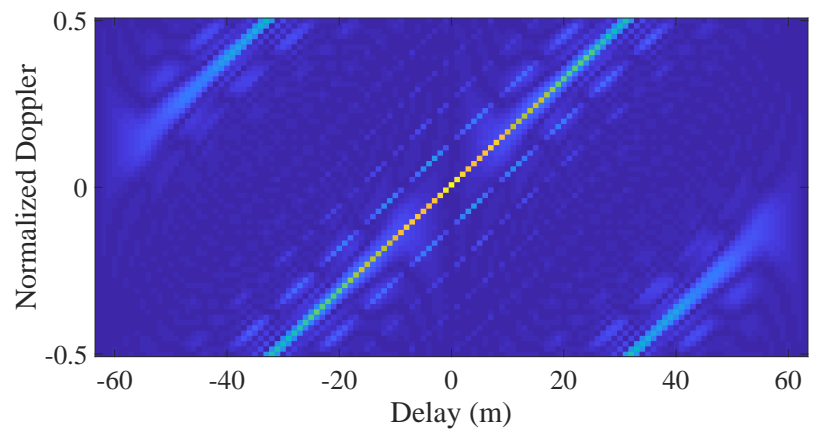

Fig. 2: Desired AF, $N=64$.

\section{PERFORMANCE ANALYSIS}

In this section, the ability of the proposed algorithms to shape radar AF is assessed. In Figure 1, the convergence behavior of the proposed continuous/discrete phase algorithms is analyzed when code length $N=$ 64. The desired $\mathrm{AF}$ in this figure is obtained from Golomb sequence, whereas the results follows similar behavior for any arbitrary AF. Notice that, since $\sum_{m=1-N}^{N-1} \sum_{\nu=0}^{N-1}|\chi(m, \nu)|^{2}=N^{3}[11]$, the definition $J(\mathrm{~dB})=10 \log _{10} \frac{J}{N^{3}}$ is used to normalize the results. As illustrated in Figure 1, the objective values decrease monotonically and converge to a stationary point, when the iteration increases. Notice that, the monotonic property of the $\mathrm{CD}$ technique along with the fact that the objective function is bounded are sufficient to prove the convergence of the proposed algorithms. It can be observed from the figure that the objective value for the discrete phase when $L=8$ is neighboring to continuous phase method. We numerically perceived that, increasing the alphabet size leads to some lower objective values, bounded with the continuous phase method. To observe the effectiveness of the proposed algorithm in shaping an $\mathrm{AF}$ we pick $\mathrm{AF}$ of the Frank sequence as the desired, and tackle $P_{\boldsymbol{x}}^{\infty}\left(P_{\boldsymbol{x}}^{L}\right)$. The AF of Frank with length $N=64$ is depicted in Figure 2. On the other hand, Figure 3 illustrates optimized AFs obtained via the proposed continuous and discrete phase algorithms. Noticing to the Figure 3a and Figure 3b, it can be observed that both continuous and discrete phase 


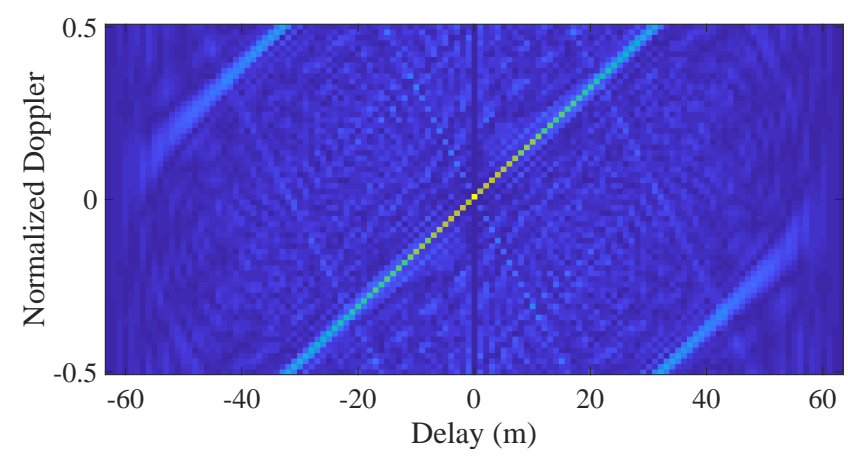

(a) Designed Discrete Phase AF, $N=64, L=4$.

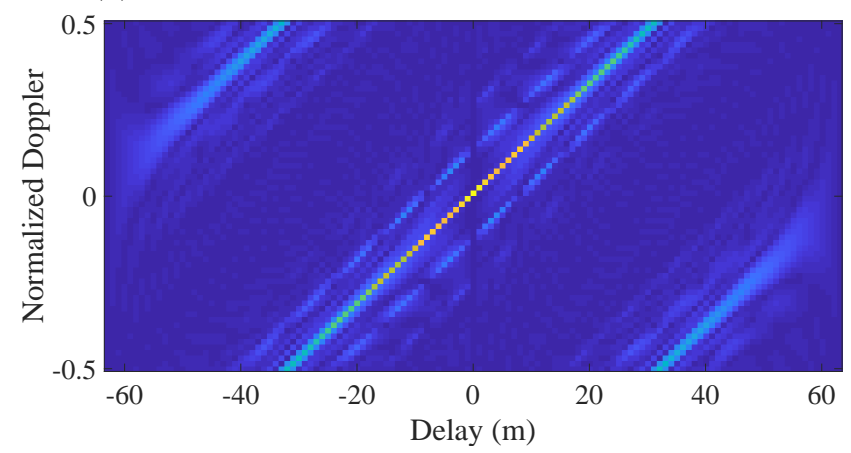

(b) Designed Continuous Phase AF, $N=64$.

Fig. 3: Designed AFs using continuous and discrete phase algorithms.

methods could effectively design sequences with the AFs similar to the desired one. Next example is depicted in Figure 4, when the designed AF is obtained with $L=8$ whereas the desired AF is taken from the chirp AF. The above two examples clearly show the powerfulness of the proposed algorithm in designing different sequences with arbitrary and Doppler tolerance AF, even with the small alphabet sizes.

\section{CONCLUSION}

In this paper, a unified framework for designing continuous/discrete phase sequences with high Doppler tolerance AF is proposed. The probing signal is obtained by synthesizing a desired AF through sequence design mini-

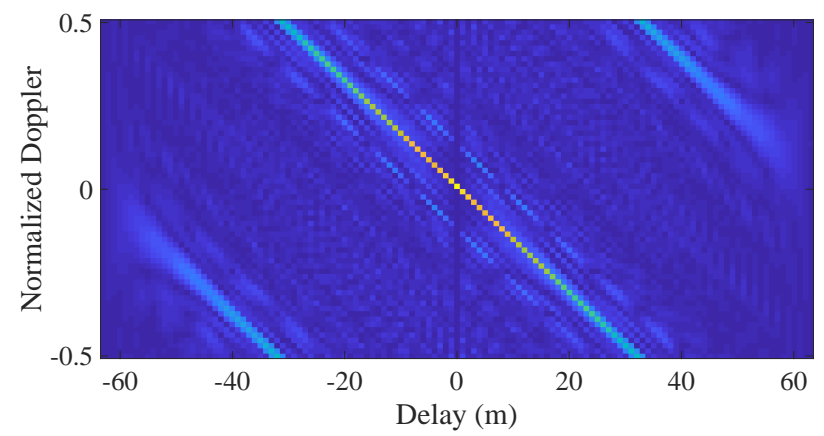

Fig. 4: Designed Discrete Phase AF, $N=64, L=8$. mizing the squared errors between the desired and realizable AF. Such sequences require simple hardware transmission modules enabling faster integration of $79 \mathrm{GHz}$ radar modules in automotive scenarios. In a future work, we will try to tackle the problem with additional constraint on the auto-correlation function of the transmitted sequences to control the range sidelobe levels.

\section{A. PROOF OF LEMMA 2.1}

Let us use the change of variable, $\phi_{d}=\arg \left(x_{d}\right)$, and define $^{5}$

$$
\begin{aligned}
& A_{m \nu}=\left|a_{m \nu}\right|^{2}+\left|b_{m \nu}\right|^{2}, \quad B_{m \nu}=a_{m \nu}^{r} b_{m \nu}^{r}+a_{m \nu}^{i} b_{m \nu}^{i}, \\
& C_{m \nu}=a_{m \nu}^{r} b_{m \nu}^{i}-a_{m \nu}^{i} b_{m \nu}^{r}, \\
& D_{m \nu}=\left|\chi_{D}(m, \nu)\right|^{4}-2\left|\chi_{D}(m, \nu)\right|^{2} A_{m \nu} .
\end{aligned}
$$

where $(.)^{r}=\Re\{()$.$\} and (.)^{i}=\Im\{()$.$\} indicate real and$ image parts, respectively. Hence, making use of the variable change $\zeta_{d}=\tan \left(\frac{\phi_{d}}{2}\right)$ and the relations $\sin \left(\phi_{d}\right)=$ $\frac{2 \tan \left(\frac{\phi_{d}}{2}\right)}{1+\tan ^{2}\left(\frac{\phi_{d}}{2}\right)}, \cos \left(\phi_{d}\right)=\frac{1-\tan ^{2}\left(\frac{\phi_{d}}{2}\right)}{1+\tan ^{2}\left(\frac{\phi_{d}}{2}\right)}[19]$, the optimization Problem $P_{d, \boldsymbol{x}^{(k)}}^{\infty}$ can be expressed as

$$
\begin{aligned}
\min _{\zeta_{d}} & \sum_{m=1-N}^{N-1} \sum_{\nu=1-N}^{N-1} D_{m \nu} \\
& +\frac{\left(\left(A_{m \nu}-2 B_{m \nu}\right) \zeta_{d}^{2}+4 C_{m \nu} \zeta_{d}+A_{m \nu}+2 B_{m \nu}\right)^{2}}{\left(\zeta_{d}^{2}+1\right)^{2}} \\
& -\frac{4\left|\chi_{D}(m, \nu)\right|^{2}\left(-B_{m \nu} \zeta_{d}^{2}+2 C_{m \nu} \zeta_{d}+B_{m \nu}\right)}{\left(\zeta_{d}^{2}+1\right)} \\
= & \frac{1}{\left(\zeta_{d}^{2}+1\right)^{2}} \sum_{m=1-N}^{N-1} \sum_{\nu=1-N}^{N-1} \\
& \left(D_{m \nu}+\left(A_{m \nu}-2 B_{m \nu}\right)^{2}+4\left|\chi_{D}(m, \nu)\right|^{2} B_{m \nu}\right) \zeta_{d}^{4} \\
& +8 C_{m \nu}\left(A_{m \nu}-2 B_{m \nu}-\left|\chi_{D}(m, \nu)\right|^{2}\right) \zeta_{d}^{3} \\
& +2\left(D_{m \nu}+8 C_{m \nu}^{2}+A_{m \nu}^{2}-4 B_{m \nu}^{2}\right) \zeta_{d}^{2} \\
& +8 C_{m \nu}\left(A_{m \nu}+2 B_{m \nu}-\left|\chi_{D}(m, \nu)\right|^{2}\right) \zeta_{d} \\
& +D_{m \nu}+\left(A_{m \nu}+2 B_{m \nu}\right)^{2}-4\left|\chi_{D}(m, \nu)\right|^{2} B_{m \nu} .
\end{aligned}
$$

Let us define the real coefficients

$$
\begin{aligned}
\alpha & =\left(D_{m \nu}+\left(A_{m \nu}-2 B_{m \nu}\right)^{2}+4\left|\chi_{D}(m, \nu)\right|^{2} B_{m \nu}\right), \\
\beta & =8 C_{m \nu}\left(A_{m \nu}-2 B_{m \nu}-\left|\chi_{D}(m, \nu)\right|^{2}\right), \\
\gamma & =2\left(D_{m \nu}+8 C_{m \nu}^{2}+A_{m \nu}^{2}-4 B_{m \nu}^{2}\right), \\
\mu & =8 C_{m \nu}\left(A_{m \nu}+2 B_{m \nu}-\left|\chi_{D}(m, \nu)\right|^{2}\right), \\
\eta & =D_{m \nu}+\left(A_{m \nu}+2 B_{m \nu}\right)^{2}-4\left|\chi_{D}(m, \nu)\right|^{2} B_{m \nu} .
\end{aligned}
$$

thus, (8) can be rewritten as

$$
P_{\zeta_{d}}^{\infty}=\left\{\min _{\zeta_{d}} \frac{\widetilde{\alpha} \zeta_{d}^{4}+\widetilde{\beta} \zeta_{d}^{3}+\widetilde{\gamma} \zeta_{d}^{2}+\widetilde{\mu} \zeta_{d}+\widetilde{\eta}}{\left(\zeta_{d}^{2}+1\right)^{2}}\right.
$$

where $\widetilde{\kappa}=\sum_{m=1-N}^{N-1} \sum_{\nu=1-N}^{N-1} \kappa$, with $\widetilde{\kappa} \in\{\widetilde{\alpha}, \widetilde{\beta}, \widetilde{\gamma}, \widetilde{\mu}, \widetilde{\eta}\}$ and $\kappa \in\{\alpha, \beta, \gamma, \mu, \eta\}$.

\footnotetext{
${ }^{5}$ For notational simplicity, herein the dependence of the coefficient $a_{m \nu}$ and $b_{m \nu}$ on step $(k)$ is implicit.
} 


\section{REFERENCES}

[1] "Texas instrument: 'MIMO radar, application report', swra554," May 2017. Available at http: //www.ti.com/lit/an/swra554a.

[2] "Ultra-small, economical and cheap radar made possible thanks to chip technology," in imec Magazine, March 2018. Available at https ://www . imec-int . com/cache/pdfs/en/ imec-magazine/imec-magazine-march-2018.

[3] H. Rohling and M. . Meinecke, "Waveform design principles for automotive radar systems," in 2001 CIE International Conference on Radar Proceedings (Cat No.01TH8559), pp. 1-4, Oct 2001.

[4] A. Bourdoux, U. Ahmad, D. Guermandi, S. Brebels, A. Dewilde, and W. V. Thillo, "PMCW waveform and MIMO technique for a $79 \mathrm{GHz}$ CMOS automotive radar," in 2016 IEEE Radar Conference (RadarConf), pp. 1-5, May 2016.

[5] F. Engels, P. Heidenreich, A. M. Zoubir, F. K. Jondral, and M. Wintermantel, "Advances in automotive radar: A framework on computationally efficient high-resolution frequency estimation," IEEE Signal Processing Magazine, vol. 34, pp. 36-46, March 2017.

[6] S. M. Patole, M. Torlak, D. Wang, and M. Ali, "Automotive radars: A review of signal processing techniques," IEEE Signal Processing Magazine, vol. 34, pp. 22-35, March 2017.

[7] M. A. Richards, J. Scheer, W. A. Holm, and W. L. Melvin, Principles of modern radar. Citeseer, 2010.

[8] M. Skolnik, Radar Handbook, Third Edition. Electronics electrical engineering, McGraw-Hill Education, 2008.

[9] N. Levanon and E. Mozeson, Radar Signals. Wiley, 2004.

[10] D. Henke, P. McCormick, S. D. Blunt, and T. Higgins, "Practical aspects of optimal mismatch filtering and adaptive pulse compression for fm waveforms," in 2015 IEEE Radar Conference (RadarCon), pp. 1149-1155, May 2015.

[11] J. Zhang, C. Shi, X. Qiu, and Y. Wu, "Shaping radar ambiguity function by $L$-phase unimodular sequence," IEEE Sensors Journal, vol. 16, pp. 56485659 , July 2016.

[12] X. Feng, Y. nan Zhao, Z. quan Zhou, and Z. feng Zhao, "Waveform design with low range sidelobe and high doppler tolerance for cognitive radar," Signal Processing, vol. 139, pp. 143 - 155, 2017.
[13] O. Aldayel, T. Guo, V. Monga, and M. Rangaswamy, "Adaptive sequential refinement: A tractable approach for ambiguity function shaping in cognitive radar," in 2017 51st Asilomar Conference on Signals, Systems, and Computers, pp. 573577 , Oct 2017.

[14] A. Aubry, A. De Maio, B. Jiang, and S. Zhang, "Ambiguity function shaping for cognitive radar via complex quartic optimization," IEEE Transactions on Signal Processing, vol. 61, pp. 5603-5619, Nov 2013.

[15] H. He, P. Stoica, and J. Li, "Designing unimodular sequence sets with good correlations; including an application to MIMO radar," IEEE Transactions on Signal Processing, vol. 57, pp. 4391-4405, Nov 2009.

[16] M. Alaee-Kerahroodi, A. Aubry, A. De Maio, M. M. Naghsh, and M. Modarres-Hashemi, "Design of binary sequences with low PSL/ISL," in 2017 25th European Signal Processing Conference (EUSIPCO), pp. 2211-2215, Aug 2017.

[17] C. Y. Chen and P. P. Vaidyanathan, "MIMO radar ambiguity properties and optimization using frequency-hopping waveforms," IEEE Transactions on Signal Processing, vol. 56, pp. 5926-5936, Dec 2008.

[18] M. Alaee-Kerahroodi, A. Aubry, A. De Maio, M. M. Naghsh, and M. Modarres-Hashemi, "A coordinatedescent framework to design low PSL/ISL sequences," IEEE Transactions on Signal Processing, vol. 65, pp. 5942-5956, Nov 2017.

[19] A. Ben-Tal and A. Nemirovski, Lectures on modern convex optimization: analysis, algorithms, and engineering applications, vol. 2. Siam, 2001. 